Please do not remove this page

RMIT

UNIVERSITY

\title{
Vehicular ad hoc networks
}

Simic, Milan

https://researchrepository.rmit.edu.au/esploro/outputs/9921859315001341/filesAndLinks?institution=61RMIT_INST\&index=null

Simic, M. (2013). Vehicular ad hoc networks. Proceedings of 201311 th International Conference on Telecommunication in Modern Satellite, Cable and Broadcasting Services (TELSIKS), 613-618.

https://doi.org/10.1109/TELSKS.2013.6704453

Document Version: Accepted Manuscript

Published Version: https://doi.org/10.1109/TELSKS.2013.6704453

Repository homepage: https://researchrepository.rmit.edu.au

(C) 2013 IEEE

Downloaded On 2023/04/27 00:29:47 +1000

Please do not remove this page 
Thank you for downloading this document from the RMIT Research Repository.

The RMIT Research Repository is an open access database showcasing the research outputs of RMIT University researchers.

RMIT Research Repository: http://researchbank.rmit.edu.au/

\section{Citation:}

Simic, M 2013, 'Vehicular ad hoc networks', in B. D. Milovanovic (ed.) Proceedings of 2013 11th International Conference on Telecommunication in Modern Satellite, Cable and Broadcasting Services (TELSIKS), Serbia, 16 - 19 October 2013, pp. 613-618.

See this record in the RMIT Research Repository at:

http://researchbank.rmit.edu.au/view/rmit:23728

Version: Accepted Manuscript

Copyright Statement: (C) 2013 IEEE

Link to Published Version:

http://dx.doi.org/10.1109/TELSKS.2013.6704453 


\title{
Vehicular Ad Hoc Networks
}

\author{
Milan N. Simic ${ }^{1}$
}

\begin{abstract}
Vehicular Ad Hoc Networks (VANETs) are computer networks of moving nodes attached to vehicles. They also include stationary infrastructure elements. This use of wireless local area network technology is becoming necessity and will grow rapidly in the near future. There is a wide range of VANET and associated Dedicated Short Range Communications (DSRC) applications. It starts from simple cases like active safety systems for driving, traffic information and collision warning, to more comprehensive Intelligent Transport Systems (ITS). Step up from simple driver assistance will be the complete takeover of the vehicle control. Finally, VANETs will support auto driver algorithms and applications for unmanned ground vehicles (UGVs). The aim of this paper is to present an overview of the technology, standards and problems, with some proposals for the quick start with the most necessary applications and for the future research.
\end{abstract}

Keywords-VANET, WLAN, DSRC, WAVE, Active Safety.

\section{INTRODUCTION}

Last two decades we have witnessed integration of computer and communications technologies [1]. In addition to that, new technology has found its way to automotive engineering, from engine control, motion control, infotainment and communications. Since there are many processing units, in an ordinary modern vehicle, we could easily say that the car is basically a "computer network on the wheels" [2]. That network, or in-vehicle domain, is further connected through on-board unit (OBU) to VANET and eventually, to Internet through roadside units (RSU).

Wireless technology has entered vehicle world long time ago, in nineteenth century. In 1898 Nikola Tesla presented his tele-operated boat at the exhibition in Madison Square Gardens [3]. Now we have wireless controlled mobile robots exploring other planets. Down on Earth, wireless communications between vehicles, Vehicle-to-Vehicle (V2V) and wireless communications vehicle-to-infrastructure (V2I) are two main subsystems of a VANET. Global Positioning System (GPS) is another vital subsystem playing important role in this ad-hoc networking. Fig.1. shows VANET components including satellites for GPS positioning and timing [4].

Infrastructure components are part of fixed, well established, networking stricture, defined by various IEEE 802 standards for wired and wireless networking. Wireless networking associated standards are available from [5].

${ }^{1}$ Milan N. Simic is with the School of Aerospace, Mechanical, and Manufacturing Engineering RMIT University, PO Box 71, Bundoora East Campus, Cnr. Plenty Road, Mc Kimmies Road, Bundoora VIC 3083, Melbourne, Australia, E-mail: milan@rmit.edu.au.

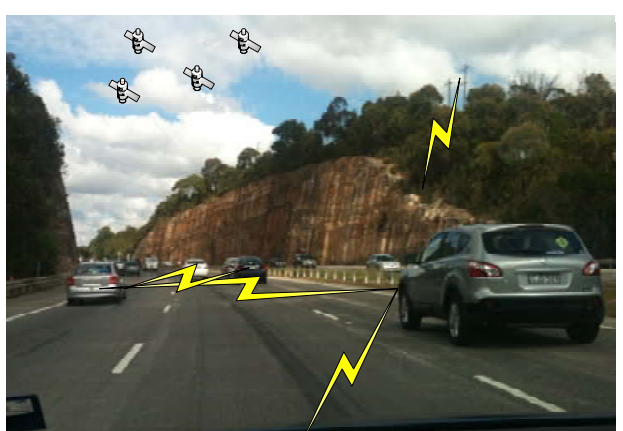

Fig.1. Networking on the road - VANET

In a VANET, as presented in Fig.1, communication devices associated to each vehicle play the role of network nodes, but also, routers and switches. Network topology changes quickly. Two nodes can communicate directly if they are within the communication range. If not, they can still establish communication in the multi-hop fashion. With the mobile telephony, we also have mobile users, or User Equipment (UE), however the network components are stationary, as shown in Fig. 2.

After this introduction, paper is structured in the following way: Analysis of some Ad-Hoc-Networking issues is given in the Section II, various applications are presented in the next section, following by study of the two most popular applications DSRC and Radio Frequency Identification (RFID). Overview of standards is in Section VI. Section VII refers to the network modelling and routing while the last section concludes with development proposals for the most urgent to implement applications.

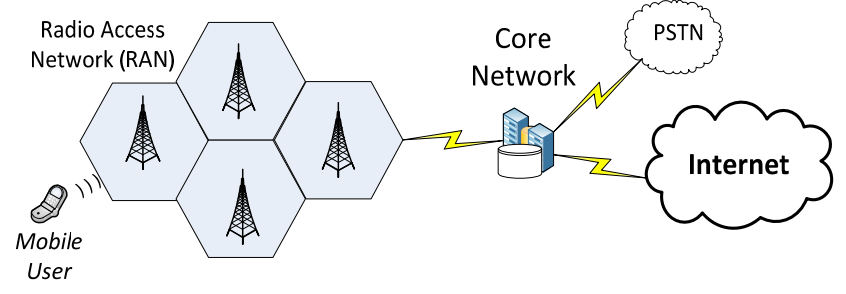

Fig.2. Mobile user and fixed communications network

\section{AD-Hoc Networking Issues}

Since VANET topology keeps changing, consequently radio propagation conditions are varying. Low antenna mountings, together with influence of moving metal objects, cause attenuation and reflections. Radio channel is untrustworthy. A network node can easily disappear from the VANET when being behind a heavy vehicle passing by. 
Network should be reliably under any environmental conditions on the road, traffic intensity and speed of vehicles.

Accordingly, Medium Access Control (MAC) plays a vital role in VANET design. Different scenarios have been investigated including Time Division Multiple Access (TDMA) in [4], Spatial Division Multiple Access (SDMA) and the most popular one, Carrier Sense Multiple Access (CSMA) as defined by IEEE 802.11.

VANET channel frequency bandwidth ranges from 10 to 20MHz. Heavy traffic may cause channel congestions, while application of multi-channels can suffer from interferences and may have synchronisation difficulties. These circumstances generate stress for the communication system. It is expensive and difficult to test communications on the road. Consequently, VANET modelling is used extensively to simulate different road scenarios and define the best VANET communications protocols [6].

There are, also issues like data security and privacy. As traffic safety will soon become more dependent on communications, data security will be a crucial issue. Like with any other digital communications, encryption techniques are employed to ensure data security on the physical layer. These techniques could be applied on data sequences, as direct spectrum, and in modulation, as frequency hopping. They scramble message, randomise carrier frequency, or time, often all of them [7]. In addition to the physical layer security methods, there are other systems used on the higher layers. Wireless Access in Vehicular Environments (WAVE) standard defines use of public key [8].

Vehicle responses to events should be based on legitimate messages, so authentication should be used in the same way as in any other networking environment. The same is with the verification of data consistency. The validity of the messages is assessed by their consistency with similar messages received recently. Network services availability is critical, suggesting that the system should be supported by alternative means of communications, like sensor based networks, or mobile phone networks.

Non-repudiation refers to the important standpoint that the sender should not be able to deny the transmission of his message. At the same time drivers privacy should be secured against unauthorised observers.

High speed mobility of the VANET nodes imposes strict, real time constraints. Consider a scenario when a vehicle is traveling at the speed of $140 \mathrm{~km} / \mathrm{h}$ and it is approaching an infrastructure, fixed unit. If reliable communication distance is $400 \mathrm{~m}$ then the time to establish and finish communication is just $20.5 \mathrm{sec}$. Standard IEEE 802.20 supports vehicular mobility with network access, at the speed of up to $250 \mathrm{Km} / \mathrm{h}$, in a MAN environment with data transfer rate of more than 1 Mbps.

\section{APPLICATIONS}

There is an increasing number of available active safety applications for drivers, while more are being researched and developed based on communications [9] and wireless sensor networks (WSN) [10]. Thanks to VANET vehicles can achieve a 360 degree awareness of the environment. Powerful
VANET and WSN integration is known as Vehicular Ad Hoc and Sensor Network (VASNET). Many safety systems are generally based on different types of sensors like radars, magnetic, laser sensors, or vision. Laser Imaging Detection and Ranging (LIDAR) is an optical remote sensing technology [11]. Radar systems use radio waves. In both cases, the distance to an object is determined by measuring the time delay between transmission of a pulse and detection of the reflected signal.

Active safety communication systems employ wireless adhoc networks and GPS to determine and maintain distance between vehicles. Other VANET /GPS applications include vehicle tracking, roadside assist tracking, navigation, geographic surveying, search and rescue operations, making precise maps and many more. With the full application of this new technology, with the integration of auto-driver algorithms, vehicle will be able to take control of itself [12], [13].

\section{DSRC}

Dedicated Short Range Communications (DSRC) technology creates more opportunities for supporting safety traffic applications, even before VANET's broad implementation. Standard specify unidirectional, or bidirectional wireless channels. DSRC systems worldwide are not compatible at the moment.

In the US standard defines a $75 \mathrm{MHz}$ licensed spectrum, in the $5.9 \mathrm{GHz}$ band, for low-latency $\mathrm{V} 2 \mathrm{~V}$ and vehicle-to infrastructure communications. While WLAN 802.11a standard supports low speed moving users, DSRC is designed to support high speeds. The DSRC band consists of seven $10 \mathrm{MHz}$ channels. The maximum DSRC data rate is $27 \mathrm{Mb} / \mathrm{s}$. Modulation used is a 64 sub-carrier Orthogonal Frequency Division Multiplexing (OFDM).

In Europe a $30 \mathrm{MHz}$ spectrum in the $5.9 \mathrm{GHz}$ band is defined for DSRC and applications like public transport management, parking management, traffic telematics and other [14]. DSRC systems across Europe are not standardised and are not used in all countries [15].

One use of DSRC for a class of vehicular safety applications is known as Cooperative Collision Warning (CCW) [16]. The goal is the prevention of vehicular collisions using $\mathrm{V} 2 \mathrm{~V}$ communications. Vehicles using $\mathrm{CCW}$ periodically broadcast short messages for the purpose of driver awareness and warning.

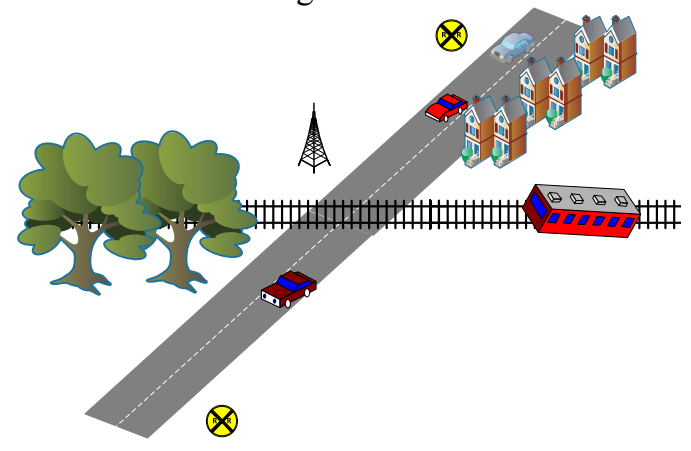

Fig.3. DSRC in level crossing application 
One of the most urgent safety applications to be fully developed, is probably level crossing. That could easily be a DSRC application of V2V and / or V2I communication. Apart from cars being equipped by communication nodes, other vehicles, like trains, or trams could be OBU equipped as well. Scenario presented in Fig. 3 shows that cars on the road have no visibility of the crossing point. Establishing communication between cars and train approaching would improve traffic safety. Radio tower, as a part of road infrastructure, could broadcast warning messages to all incoming vehicles.

Communication systems also take the role of route guidance, adjustable speed control, cooperative driving and other.

\section{RFID}

RFID is an emerging radio technology already used for remote data acquisition. Automatic Identification and Data Capture (AIDC), modern manufacturing automation and many other applications are based on RF identification. An RFID system consists of RFID tag and RFID reader. It is a master-slave configuration. Reading device could be directly connected to the data processing system, or could store data and upload it to the system in a batch mode of operation. There is no worldwide standard for RFID applications. When an automatic payment and traffic control system is applied every vehicle on the road must have an electronics tag, which is a radio frequency identification device.

RFID receivers read tags within the specified range, which could be up to $60 \mathrm{~m}$ away, or beyond the line of sight. Tags, that store data, can be attached to various objects. Products, animals, or even people could be tagged.

An RFID tag has two parts: antenna as a coupling element and an integrated circuit (IC). IC is storing and processing data, as well as, performing communication tasks, i.e. RF modulation and demodulation. According to the power requirements, tags could be passive, active and semi-passive. Passive tags have no battery attached and receive energy for its operation just from the reader.

Antenna selection depends on applications and operating frequency. Low frequency, passive tags are inductively coupled. According to the Faraday's law of electromagnetic induction, electromotive force, E, induced in a coil, when magnetic flux, $\Phi$, changes is given as:

$$
E=-N \frac{d \Phi}{d t}
$$

- If the frequency of the change is low (LF), $\mathrm{N}$ should be larger, i.e. more coil turns are needed to achieve usable voltage level for correct IC operation.

- When high frequency (HF) is used, as $13.56 \mathrm{MHz}$, less coil turns are needed and tags are smaller.

- When ultra-high frequency (UHF) is used, in the range of $300 \mathrm{MHz}$ to $3000 \mathrm{MHz}$, or in the microwave range $(300 \mathrm{MHz}-300 \mathrm{GHz})$, passive tags can used with dipole antennas.
RFID application for automatic vehicle identification is using frequencies in the range of $2.446-2.454 \mathrm{GHz}$. Just this relatively simple application, i.e. transition to automatic payment system saves energy, time, and congestions on the road, and proves to be environmental friendly by eliminating queues at the toll payment stations.

\section{WAVE STANDARDS}

VANET get access to Internet through infrastructure elements, where RSUs act as routers. WAVE supports integration of ad hoc and infrastructure networks. An approach in IPv6 auto-configuration VANET cross layer design, based on IEEE 1609, is given in [17]. Wireless Local Area Network, IEEE 802.11 standard is extended with IEEE $802.11 \mathrm{p}$ to cover WAVE technology. It takes care of data link and physical layers, as shown in Fig. 4. The figure presents the roles of IEEE 802 and IEEE 1609.

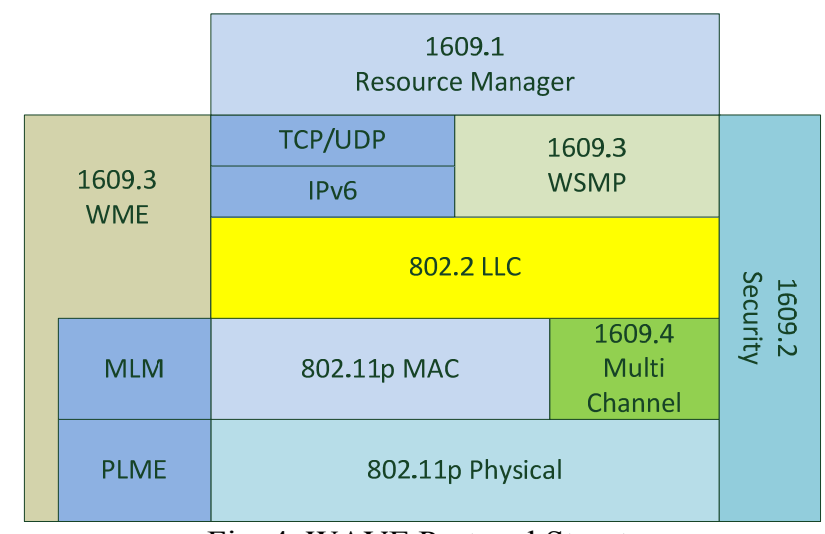

Fig. 4. WAVE Protocol Structure

The main goal of the IEEE 802.11p Amendment is to upgrade 802.11 to support vehicle mobility of up to $150 \mathrm{~km} / \mathrm{hr}$. Frame format is altered and delay spread tolerance increased. The channel bandwidth is defined from $20 \mathrm{MHz}$ in $802.11 \mathrm{a}$ to $10 \mathrm{MHz}$ in $802.11 \mathrm{p}$. Upper protocol layers, as defined by OSI architecture, are covered by IEEE 1609 specifications. Four 1609 sub-groups are defined as:

1. IEEE1609.1, Resource Manager, Upper layers

2. IEEE1609.2, WAVE Security services

3. IEEE1609.3, Network layer; WAVE short message protocol (WSMP) is responsible for addressing and routing. It ensures that communication system supports two protocols stacks. They are TCP/IPv6 and WSMP as show in Fig. 4.

4. IEEE1609.4 is defined for multi-channel operations. Together with IEEE802.11p it takes care of the lower layers. This protocol defines a time-division scheme for DSRC radios and subsequently supports different applications concurrently.

Since synchronisation, among all devices, is extremely important, timing is usually obtained through GPS signalling. It is shown in [18] that time division switching does not guarantee the most efficient channel utilisation.

More detail descriptions of the WAVE architecture can be found in [19], [20], [21] and of course in [5]. 


\section{NETwORK MODELLING AND ROUTING}

VANET is just a special case of Mobile Ad-hoc Networks (MANETs). The differences between VANET and MANET are that, in VANET restrictions over movement are defined by road layout, topology and traffic rules. We could say that VANET topology is not absolutely random. Following that, for the research purposes, it is logical to use accurate simulations based on real road maps. In realistic models, cars are not moving completely ad-hoc. They are constrained by streets, traffic rules, objects and crossroads. In the heavily populated areas, objects such as buildings, reduce signal strength and can influence communications and routing.

The main data transport mechanism in VANET is packet forwarding to the neighbours. Each neighbour is forwarding further. Therefore, nodes could be connected to the ad-hoc network anywhere. Cars, currently located in a geographic region, form an ad-hoc network. This network can support traffic control, monitoring and safety. Cars can receive messages of suddenly changed traffic conditions, or accident warnings.

GPS plays important role in positioning and timing. A study of the GPS position errors in VANETs and their impact on a real-world accident warning system is given in [22]. It is shown, by experimentation, that the average GPS errors in the range of 4-6m do not have negative effect on the VANET accident warning system. The authors have implemented the idea presented in [23] to deliver alert messages requiring minimum number of transmissions, or hops executed by the message.

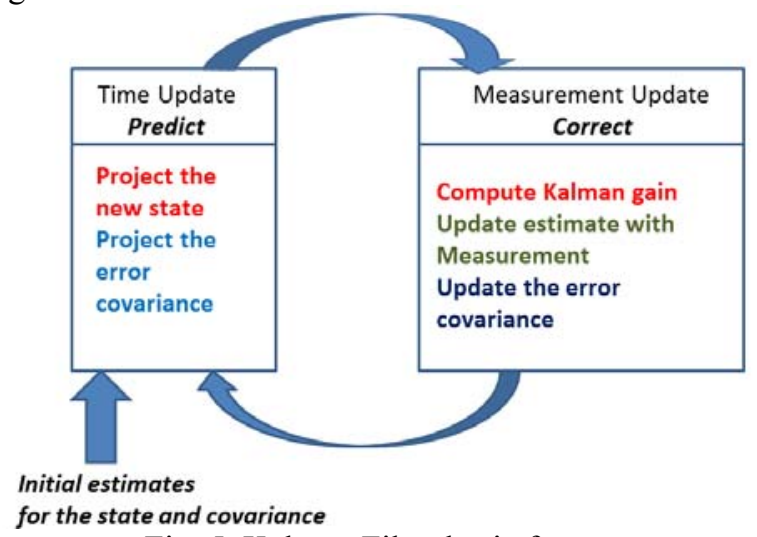

Fig. 5. Kalman Filter basic form

For other applications, where more precise positioning is requested, we would recommend applications of localization and mapping methodology as applied in mobile robotics. Kalman filter, as shown in Fig. 5. is one of the most popular tools for the location predictions. Position estimate relies on additional measurements, apart from GPS. They could be obtained by odometer, compass, radar, laser or other sensors. Neighbourhood awareness is important for safety VANET applications. A secure cooperative approach for nonline-ofsight location verification in VANET is given in [24]. A comprehensive data fusion model is presented, which includes GPS data, ad-hoc information, dead reckoning and map matching with the use of statistical filters. That approach improves neighbourhood awareness under non-line of sight (NLOS) conditions.

Mobility modelling is not the same for the city and outside of the high populated areas. We could classify routing protocols as Rural, Urban and Freeway types. In the rural scenarios we have low network density while on the freeway we have high speed moving vehicles. A survey of routing protocols for VANET in urban environment is given in [25]. Performance analysis of XOR-based routing in urban scenarios is presented in [26]. This is an interesting approach since it does not depend on information about the network topology, or the position of the nodes in order to perform traffic forwarding. Scenario for building routing tables is given in Fig. 6.

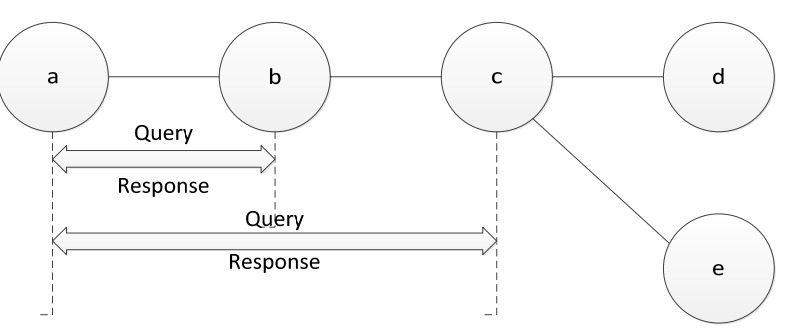

Fig. 6. Building routing table trough Query / Response

More on Urban routing protocols could be found in [27, 28]. Rural VANET scenario is covered in [29] with references to highway as well. Network modelling and nodes mobility are still the key areas of VANET research. Many mobility models are based on random waypoint modelling and effects on routing performance. Realistic mobility model for vehicular network is critical for accurate simulation. Consequently, the Street Random Waypoint (STRAW) mobility model is used for VANET mobility simulation, which constrains node movement to the streets, and limits node mobility according to the vehicular congestion and traffic control mechanisms. Using of the real maps, for VANET modelling, results in the better simulation in comparison to a general random waypoint modelling, which is more appropriate for MANET. More on random waypoint mobility modelling can be found in [30-32].

A statistical channel model for VANET simulation based on measurement data is given in [6]. One of the conclusions and suggestions for the future work is to extract the parameters for fading distributions from the real data.

A performance evaluation of mobility modelling and routing protocols for inter vehicular communication system is given in [33]. A survey of position based VANET routing protocols is presented in [34]. Last decade we have witnessed increasing activities of automotive industry, other local and international stakeholders in the area of Car2Car communications. European Commission, with a large number of other parties, has established eSafety Working Group in 2001 [35]. In addition to that Car2Car Communication Consortium was formed by few largest car manufacturers. Following all of that, there were number large $\mathrm{C} 2 \mathrm{C}$ projects that advanced research in this area. One of the most promising was GeoNet project run from 2008 to 2010. The main idea was integration of Inter-Vehicle Communication (IVC) to IPV6 structure. Although that many questions were 
left unanswered specifications created by GeoNet project were assimilated by European Telecommunications Standards Institute (ETSI). Among open questions for the further research we have congestion control, multi-radio support, transport layer, IPv6 encoding of GeoDestinations, IPv6 addresses mapping to GeoNet addresses and protocols for GeoRouting.

There are number of IVC routing protocols, which can be categorised based on different criteria. Based on communication model we could have following categories: General Information services, Vehicle safety information services, Individual motion control and Group motion control. Based on the addressing requirements IVC routing protocols could be characterized as unicast, with fixed or with geographical addresses, multicast with geographical addresses and routing protocols that apply diffusion. Flooding based protocols, then cluster based protocol and similar are regarded here. A cluster, as show in Fig. 7, consists of group of neighbourhood nodes grouped according to earlier defined rules. Rules are defined by applied algorithm. Each cluster has a cluster head selected by other nodes. Each node could be cluster head unless they are different. For example, nodes with $3 \mathrm{G}$ network connectivity may have priority. i.e. be more suitable for $\mathrm{CH}$ role.

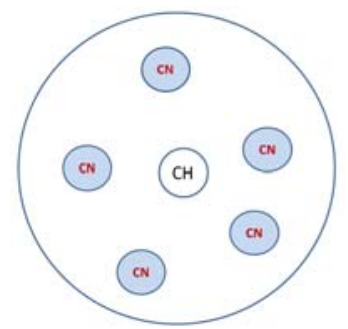

Fig. 7. A cluster with cluster head and cluster nodes

Clustering is expected to give contribution to more reliable and scalable networking. A survey on clustering based algorithms is given in [35]. Like with most of the other routing protocols' approaches, real life experiments and testing, on the road, are big challenge. Another simple classification could be as following: Flooding routing protocols, Directed flooding and Nonflooding. Sometimes routing protocols are not categorised based on the communication algorithms applied. Instead of that performance criteria are analysed, as usually done in communications and computer science / operating systems. Performance criteria are basically delivery rate, latency, packet overhead, or similar. Protocols could also be classified based on routing matrix or other criteria. A comprehensive review of IVC routing protocols is given in [35].

\section{CONCLUSION AND PROPOSALS}

This paper is a short review of a relatively new area of wireless networking. There are still lot of research challenges to fulfil requirements, as defined by IEEE standards, but the basic technology is already available. Economic and environmental benefits for the community highly overcome investment in the further research and technology implementation. Finally this will also lead to integration of wireless communications, robotics and automotive engineering. Although a lot of simulations and testing of VANET protocols and standards are needed, there are many applications that could be used now.

- One, instant proposal would be to urgently develop safety applications for the level crossing, worldwide.

- As already mentioned, just transition to automatic freeway payment system (standardised) saves energy, time, congestions on the road and it is ecological friendly.

- A mobile user in Fig. 2 could be in a car from Fig. 1. Sharing data among two networks is one of the new research proposals and challenges.

- IPv6 encoding, with 128 bits, has enough address space to include localisation in a comprehensive networking.

VANET technologies will further enhance fuel economy, road safety, sustainability and environmental protection.

\section{REFERENCES}

[1] M. Simic, A. Sharma, R. Meyer, K. Cheng, N. Dikic, R. Humphris, et al., Contemporary Digital Radio Technologies. Melbourne: RMIT Print, 2006.

[2] M. Simic, "Car Internet and Ethernet Networking System," in XXI International Conference "Science and Motor Vehicles”, Belgrade, 2007.

[3] J. J. Glenn, The Complete Patents of Nikola Tesla vol. 1. New York: Barnes \& Noble Books, 1994.

[4] M. S. Almalag, S. Olariu, and M. C. Weigle, "TDMA cluster-based MAC for VANETs (TCMAC)," in World of Wireless, Mobile and Multimedia Networks (WoWMoM), 2012 IEEE International Symposium on a, 2012, pp. 1-6.

[5] IEEE. (2013, 19.05.2013). IEEE Standards. Available:

http://standards.ieee.org/getieee802/index.html

[6] A. Cardote, F. Neves, S. Sargento, and P. Steenkiste, "A statistical channel model for realistic simulation in VANET," in Vehicular Networking Conference (VNC), 2012 IEEE, 2012, pp. 48-55.

[7] N. I. Shuhaimi and T. Juhana, "Security in vehicular ad-hoc network with Identity-Based Cryptography approach: A survey," in Telecommunication Systems, Services, and Applications (TSSA), 2012 7th International Conference on, 2012, pp. 276-279.

[8] M. Gerlach, "Trust for Vehicular Applications," in Autonomous Decentralized Systems, 2007. ISADS '07. Eighth International Symposium on, 2007, pp. 295-304.

[9] M. Gerlach, S. Steglich, S. Arbanowski, M. Wegdam, and H. Teunissen, "Trustworthy applications for Vehicular Environments," Vehicular Technology Magazine, IEEE, vol. 1, pp. 9-15, 2006.

[10] M. J. Piran, G. R. Murthy, G. P. Babu, and E. Ahvar, "Total GPS-free Localization Protocol for Vehicular Ad Hoc and Sensor Networks (VASNET)," in Computational Intelligence, Modelling and Simulation (CIMSiM), 2011 Third International Conference on, 2011, pp. 388-393. 
[11] L. Gaung and M. Tomizuka, "LIDAR Sensing for Vehicle Lateral Guidance: Algorithm and Experimental Study," Mechatronics, IEEE/ASME Transactions on, vol. 11, pp. 653-660, 2006.

[12] R. N. Jazar, M. Simic, and A. Khazaei, "Autodriver Algorithm for Autonomous Vehicle," in 2010 ASME International Mechanical Engineering Congress and $R \& D$ Expo, Vancouver, British Columbia, Canada 2010.

[13] A. Bourmistrova, M. Simic, R. Hoseinnezhad, and R. N. Jazar, "Autodriver algorithm," Journal of Systemics, Cybernetics and Informatics, vol. 9, p. 8, 2011.

[14] B. R. Franciscatto, A. C. Souza, C. Defay, T. T. Trang, and T. P. Vuong, "High gain microstrip patch antenna array using multiple superstrate layers for DSRC applications," in Antennas and Propagation in Wireless Communications (APWC), 2012 IEEEAPS Topical Conference on, 2012, pp. 736-739.

[15] ETSI, "http://www.etsi.org/standards."

[16] T. ElBatt, et al., "Cooperative Collision Warning Using Dedicated Short Range Wireless Communications," in VANET'06, Los Angeles, California, USA, 2006.

[17] C. S. Li and H. C. Chao, "IPv6 auto-configuration VANET cross layer design based on IEEE 1609," Networks, IET, vol. 1, pp. 199-206, 2012.

[18] W. Qing, L. Supeng, F. Huirong, and Z. Yan, "An IEEE 802.11p-Based Multichannel MAC Scheme With Channel Coordination for Vehicular Ad Hoc Networks," Intelligent Transportation Systems, IEEE Transactions on, vol. 13, pp. 449-458, 2012.

[19] M. A. Abakar, R. A. Saeed, A. A. Hassan, O. M. Mohammed, O. Khalifa, and S. Islam, "The challenges of wireless internet access in vehicular environments," in Information and Communication Technology for the Muslim World (ICT4M), 2010 International Conference on, 2010, pp. D31-D36.

[20] A. Jafari, S. Al-Khayatt, and A. Dogman, "Performance evaluation of IEEE 802.11p for vehicular communication networks," in Communication Systems, Networks \& Digital Signal Processing (CSNDSP), 2012 8th International Symposium on, 2012, pp. 1-5.

[21] H. Hartenstein and K. P. Laberteaux, "A tutorial survey on vehicular ad hoc networks," Communications Magazine, IEEE, vol. 46, pp. 164171, 2008.

[22] A. Amoroso, G. Marfia, and M. Roccetti, "GPS position errors in VANETS: Their impact on a realworld accident warning system," in Satellite Telecommunications (ESTEL), 2012 IEEE First AESS European Conference on, 2012, pp. 1-5.

[23] M. Roccetti, G. Marfia, and A. Amoroso, "An optimal 1D vehicular accidentwarning algorithm for realistic scenarios," in Computers and Communications (ISCC), 2010 IEEE Symposium on, 2010, pp. 145-150.
[24] O. Abumansoor and A. Boukerche, "A Secure Cooperative Approach for Nonline-of-Sight Location Verification in VANET," Vehicular Technology, IEEE Transactions on, vol. 61, pp. 275285, 2012.

[25] P. S. Nithya Darisini and N. S. Kumari, "A survey of routing protocols for VANET in urban scenarios," in Pattern Recognition, Informatics and Medical Engineering (PRIME), $2013 \quad$ International Conference on, 2013, pp. 464-467.

[26] E. P. F. Cruz, C. A. V. Campos, R. Pasquini, L. F. Faina, and R. Oliveira, "Performance analysis of XOR-based routing in urban vehicular ad hoc networks," in Wireless Communications and Networking Conference (WCNC), 2012 IEEE, 2012, pp. 2521-2525.

[27] C. Yufeng, X. Zhengtao, J. Wei, and J. Weirong, "An adaptive cross-layer multi-path routing protocol for urban VANET," in Automation and Logistics (ICAL), 2010 IEEE International Conference on, 2010, pp. 603-608.

[28] L. Jie, G. Xinxing, Z. Tong, and Y. Wei, "A Mobile Infrastructure Based VANET Routing Protocol in the Urban Environment," in Communications and Mobile Computing (CMC), 2010 International Conference on, 2010, pp. 432-437.

[29] M. Zhang and R. S. Wolff, "Routing protocols for vehicular Ad Hoc networks in rural areas," Communications Magazine, IEEE, vol. 46, pp. 126131, 2008.

[30] M. S. Hossain and M. Atiquzzaman, "Stochastic Properties and Application of City Section Mobility Model," in Global Telecommunications Conference, 2009. GLOBECOM 2009. IEEE, 2009, pp. 1-6.

[31] R. Baumann, S. Heimlicher, and M. May, "Towards Realistic Mobility Models for Vehicular Ad-hoc Networks," in 2007 Mobile Networking for Vehicular Environments, 2007, pp. 73-78.

[32] A. R. Momen, A. S. Hassani, A. Mirzaee, and P. Azmi, "Intelligent Vehicle Mobility Modeling Based on a Sub-Optimal Path Finding Method," in Vehicular Technology Conference, 2006. VTC 2006Spring. IEEE 63rd, 2006, pp. 3012-3015.

[33] R. S. Shukla and N. Tyagi, "Performance evaluation of mobility model and routing protocols for inter vehicular communication system," in Emerging Trends in Networks and Computer Communications (ETNCC), 2011 International Conference on, 2011, pp. 263-266.

[34] C. A. T. H. Tee and A. C. R. Lee, "Survey of position based routing for Inter Vehicle Communication system," in Distributed Framework and Applications, 2008. DFmA 2008. First International Conference on, 2008, pp. 174-182.

[35] Z. C. Taysi and A. G. Yavuz, "Routing Protocols for GeoNet: A Survey," Intelligent Transportation Systems, IEEE Transactions on, vol. 13, pp. 939954, 2012. 